\title{
Focal Cortical Thickening along the Lateral Aspect of the Proximal Femoral Shaft: A Warning Radiographic Sign for Subsequent Complete Atypical Femoral Fracture in Patients on Bisphosphonates
}

\author{
${ }^{1}$ Sachin Agrawal, ${ }^{2}$ Amelia Santosa
}

\begin{abstract}
Atypical femoral fracture is a known complication of patients receiving long-term bisphosphonates for osteoporosis. Recognizing early radiographic features is important to provide timely care and management before the development of a more debilitating complete fracture with its associated morbidity and mortality. We report a case of a 72-year-old lady on long-term treatment with alendronate, who presented initially with groin pain and subsequently with a femoral fracture, without any major trauma. The initial radiographic finding of focal thickening along the lateral cortex of the proximal femur was missed.
\end{abstract}

Keywords: Atypical femoral fracture, Bisphosphonates, Focal cortical thickening.

How to cite this article: Agrawal S, Santosa A. Focal Cortical Thickening along the Lateral Aspect of the Proximal Femoral Shaft: A Warning Radiographic Sign for Subsequent Complete Atypical Femoral Fracture in Patients on Bisphosphonates. Int J Recent Surg Med Sci 2017;3(1):58-60.

Source of support: Nil

Conflict of interest: None

\section{INTRODUCTION}

Bisphosphonates are widely used for the treatment of patients with osteoporosis. They reduce the incidence of vertebral and other fractures by suppressing the loss of bone volume. ${ }^{1}$ Contradictorily, however, long-term biphosphonate usage is itself sometimes associated with increased incidence of atypical fractures. These biphosphonate-related atypical fractures were first described in 2005 by Odvina et al. ${ }^{2}$ These fractures are insufficiency fractures with characteristic location and radiographic

\footnotetext{
${ }^{1,2}$ Consultant

${ }^{1}$ Department of Radiodiagnosis, National University Hospital Singapore

${ }^{2}$ Department of Rheumatology, National University Hospital Singapore

Corresponding Author: Sachin Agrawal, Consultant, Department of Radiodiagnosis, National University Hospital, Singapore Phone: +006590063238, e-mail: sachin_nandkishore_agrawal@ nuhs.edu.sg
}

features. ${ }^{3}$ There are few reports in the radiology literature and many physicians including radiologists are not aware of this entity. Here, we report a patient with a biphosphonate-related fracture. The clinical presentation, characteristic radiographic features, further imaging work-up, and differential diagnosis and management strategies have been discussed with emphasis on early radiographic signs before the development of a complete fracture.

\section{CASE REPORT}

A 76-year-old woman was on long-term treatment with alendronate for osteoporosis. She presented to the outpatient with a 1-week history of groin pain. Premorbidly, she was ambulant with a walking stick. Since 1 week, pain has affected her mobility and she has become unsteady with the walking stick. A radiograph (Fig. 1) was performed which was read as normal by the reporting radiologist and the patient was discharged.

A few hours later on the same day, the patient presented again with markedly increased pain after she tripped and fell in the kitchen. She was unable to bear her weight and walk after the incident. A repeated

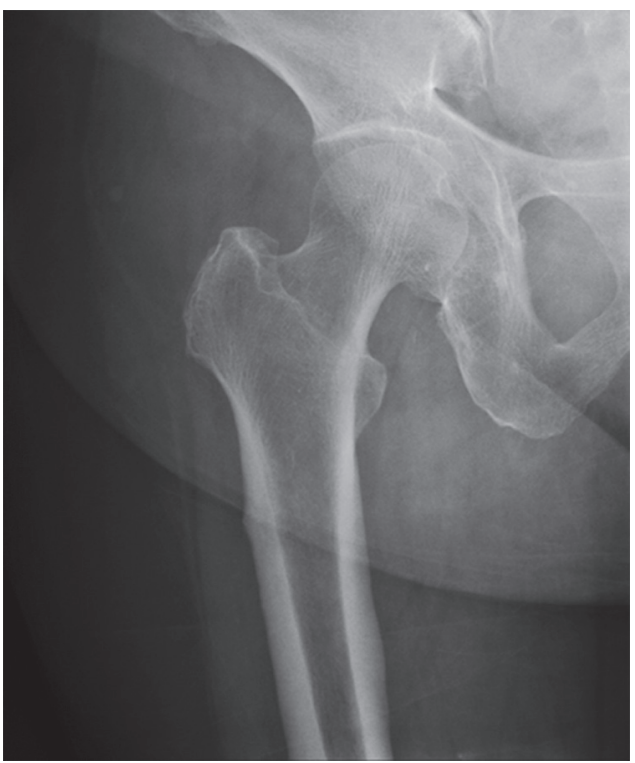

Fig. 1: Frontal radiograph of the right hip joint (on initial presentation) was read as showing no fracture or dislocation 


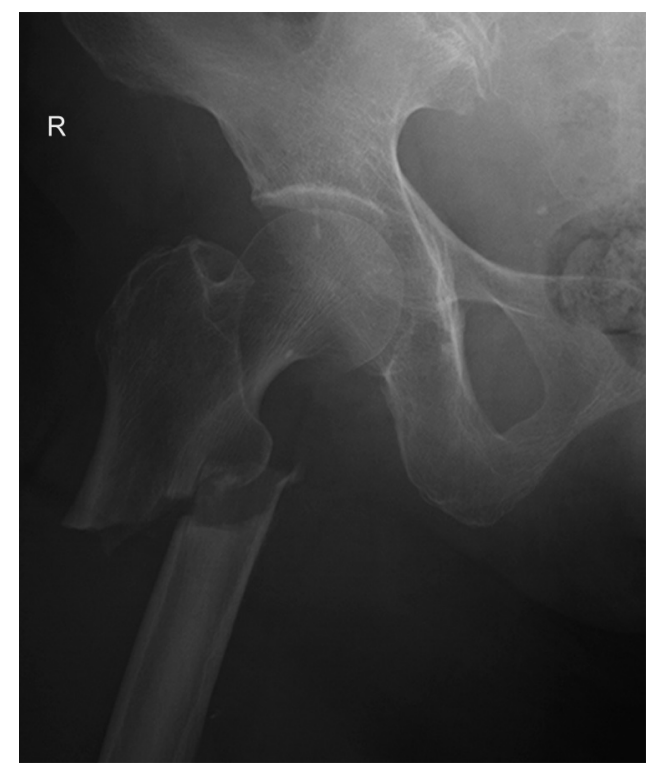

Fig. 2: Frontal radiograph of the right hip joint on follow-up demonstrates a displaced and complete transverse fracture through the proximal shaft of the femur (note the presence of a medial spike)

radiograph showed a complete and displaced transverse fracture through the proximal shaft of the femur (Fig. 2). No underlying pathological lesion was identified. A diagnosis of insufficiency fracture was made based on the transverse orientation of the fracture and absence of an underlying pathological lesion. The patient was managed with internal fixation of the fracture.

A retrospective review of the earlier radiograph revealed presence of focal cortical thickening along the lateral cortex of the proximal femur (Fig. 3), the site of later development of a complete fracture.

\section{DISCUSSION}

Atypical femoral fracture (AFF) is an insufficiency fracture that occurs as a result of normal activity or low-energy trauma in bones that are weakened by a generalized process, such as osteoporosis. Insufficiency fractures of the proximal femoral shaft are quite rare in the osteoporotic population. ${ }^{4}$ Several studies have identified a strong relationship between long-term receipt of bisphosphonates and atypical fractures in the proximal femoral shaft. ${ }^{5}$ It is rather a paradox that a fracture would be associated with biphosphonate therapy, as they are prescribed to reduce the incidence of osteoporosis-related fractures. The exact mechanism for biphosphonate-related insufficiency femoral fracture is not entirely understood, although it has been suggested that suppression of bone turnover could cause microdamage accumulation that allows small cracks to progress. ${ }^{6}$

The American Society for Bone and Mineral Research (ASBMR) task force has summarized the published

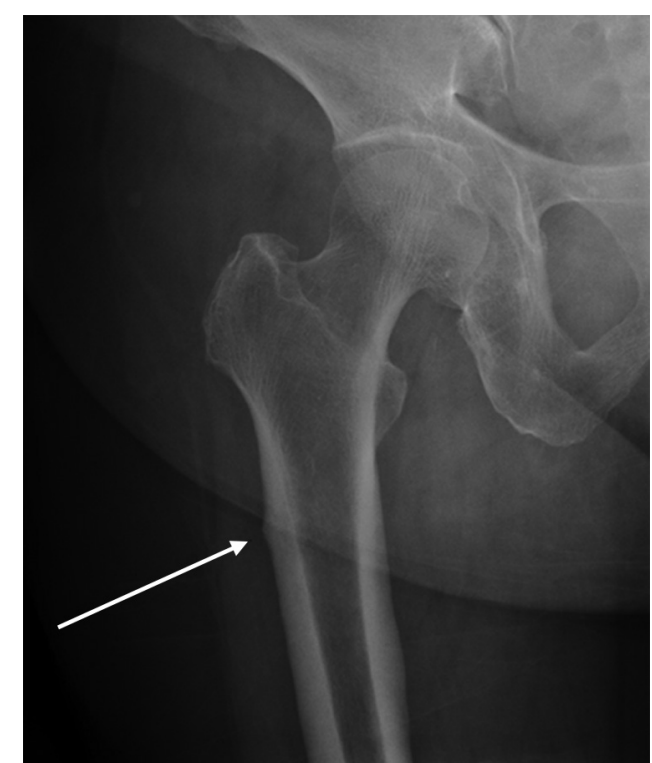

Fig. 3: A retrospective review of the frontal radiograph of the right hip joint (at initial presentation) showed the presence of focal cortical thickening along the lateral cortex of the proximal femur, the site of later development of a complete fracture

reports on AFFs and defined the features of these fractures. ${ }^{7}$ In 2013, the case definition was revised by the ASBMR to highlight radiographic features that distinguish AFFs from ordinary osteoporotic femoral diaphyseal fractures (Table 1). ${ }^{8}$ Localized periosteal reaction or endosteal thickening of the lateral cortex was included in the revised case definition for the first time. This focal thickening of the lateral femoral cortex was present in our patient in the earlier radiograph, although was missed.

As per the ASBMR task force's recommendations, an AFF usually begins as a small focal beak-like cortical thickening of the lateral cortex which is usually the result of focal periosteal/endosteal thickening related to a microfracture. Subsequently, there may be an appearance of a small incomplete lucent fracture line in the region of the periosteal thickening which may later progress to a complete fracture involving both the lateral and the medial cortex. ${ }^{8}$ In the early stage, patients can present with groin or thigh pain which can be misdiagnosed to degenerative disease of the lumbar spine and sacroiliac joints. This prodromal thigh or groin pain was observed in approximately $70 \%$ of patients. ${ }^{1}$ The contralateral femur may be involved in approximately 20 to $64 \%$ of cases. ${ }^{5}$

Thus, if a patient on biphosphonate therapy presents with groin pain, one should have a high index of suspicion for a possible $\mathrm{AFF}$, and a radiograph should be done. Also, if a radiologist encounters a radiograph of a patient with groin or thigh pain, he or she should look for signs of focal cortical thickening and sought the clinical history of biphosphonate therapy. Focal cortical thickening should 
Table 1: The ASBMR task force 2013 revised case definition of AFFs

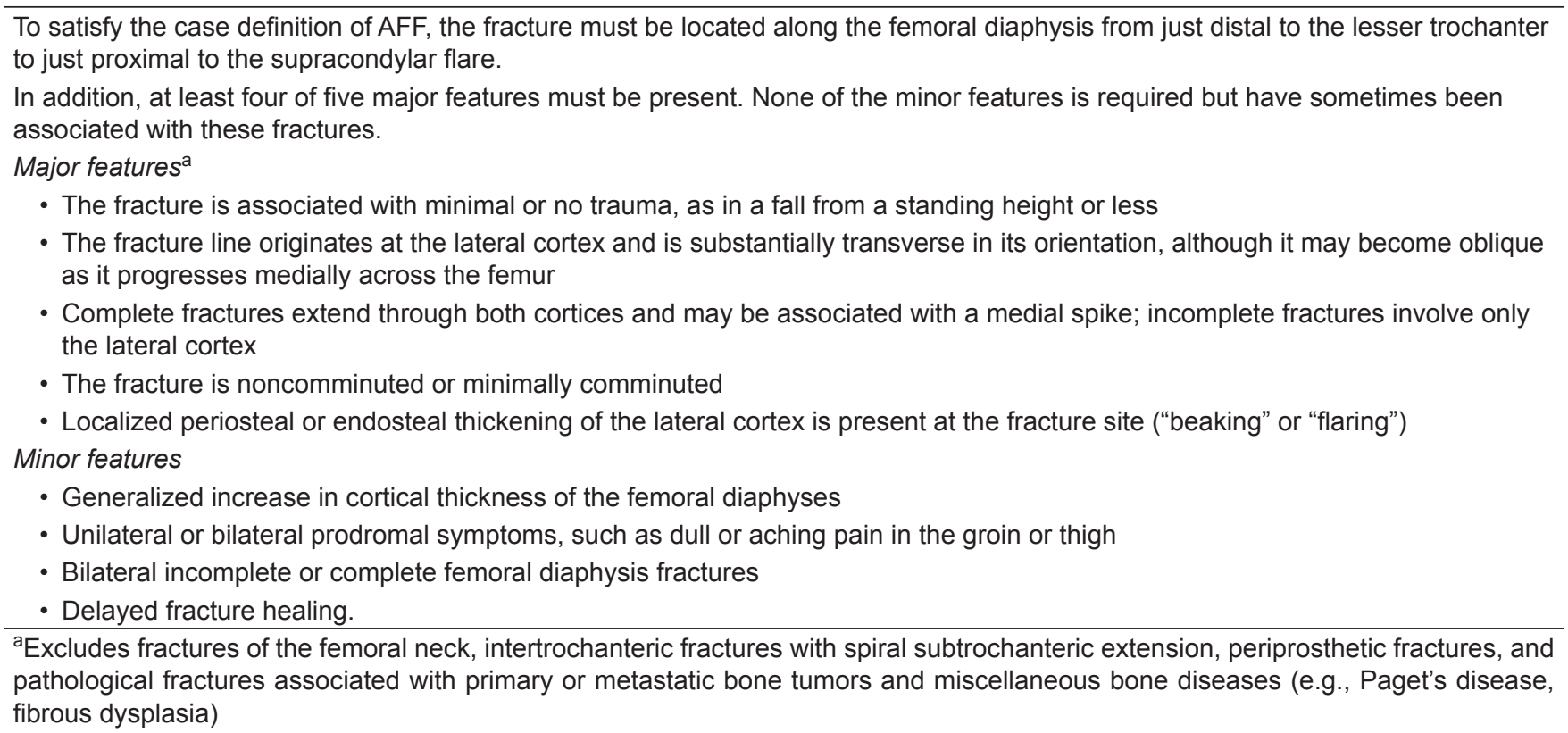

be further evaluated with magnetic resonance imaging or a bone scan. ${ }^{4}$ The bone scan would demonstrate a focus of increased uptake in the lateral cortex of the proximal femur. Magnetic resonance imaging scan would reveal focal thickening of the lateral cortex with a focus marrow edema with or without the presence of an incomplete fracture line. These findings are suggestive of a microfracture and associated stress response. Management strategies include limited weight-bearing through the use of crutches or a walker when there is focal cortical thickening, and prophylactic intramedullary nail fixation if a small incomplete fracture line is seen. ${ }^{8}$

Fractures in the proximal femoral diaphysis seen in the setting of low-energy trauma can occur in other clinical situations. These include stress fractures in young military recruits and athletes, pseudofractures of osteomalacia, and pathologic fractures. These fractures can be differentiated based on their clinical presentation and imaging features. Both the stress fractures, as well as the pseudofractures of osteomalacia, involve the medial cortex, as opposed to the biphosphonate-related AFFs which involve the lateral cortex. Pathologic fractures usually demonstrate features of underlying disease like metastases and Paget's disease. ${ }^{5}$

\section{CONCLUSION}

Focal cortical thickening of the lateral cortex of the femoral shaft serves as a warning sign for the subsequent development of a complete AFF in patients on long-term biphosphonate therapy. Awareness and recognition of this early radiographic sign, especially in patients presenting with groin or thigh pain, allows for the institution of appropriate early intervention before the development of a debilitating complication.

\section{REFERENCES}

1. Saita $Y$, Ishijima M, Kaneko K. Atypical femoral fractures and bisphosphonate use: current evidence and clinical implications. Ther Adv Chronic Dis 2015 Jul;6(4):185-193.

2. Odvina CV, Zerwekh JE, Rao DS, Maalouf N, Gottschalk FA, Pak CY. Severely suppressed bone turnover: a potential complication of alendronate therapy. J Clin Endocrinol Metab 2005 Mar;90(3):1294-1301.

3. Toro G, Ojeda-Thies C, Calabrò G, Taro G, Moretti A, Guerra GM, Caba-Doussoux P, Iolascon G. Management of atypical femoral fracture: a scoping review and comprehensive algorithm. BMC Musculoskelet Disord 2016 May 23; 17:227.

4. Bush LA, Chew FS. Subtrochanteric femoral insufficiency fracture following bisphosphonate therapy for osseous metastases. Radiol Case Rep 2015 Dec;3(4):232.

5. Porrino JA, Kohl CA, Taljanovic M, Rogers LF. Diagnosis of proximal femoral insufficiency fractures in patients receiving bisphosphonate therapy. AJR Am J Roentgenol 2010 Apr;194(4):1061-1064.

6. Donnelly E, Saleh A, Unnanuntana A, Lane JM. Atypical femoral fractures: epidemiology, etiology, and patient management. Curr Opin Support Palliat Care 2012 Sep;6(3): 348-354.

7. Shane E, Burr D, Ebeling PR, Abrahamsen B, Adler RA, Brown TD, Cheung AM, Cosman F, Curtis JR, Dell R, et al. Atypical subtrochanteric and diaphyseal femoral fractures: report of a task force of the American Society for Bone and Mineral Research. J Bone Miner Res 2010 Nov;25(11):2267-2294.

8. Shane E, Burr D, Abrahamsen B, Adler RA, Brown TD, Cheung AM, Cosman F, Curtis JR, Dell R, Dempster DW, et al. Atypical subtrochanteric and diaphyseal femoral fractures: second report of a task force of the American Society for Bone and Mineral Research. J Bone Miner Res 2014 Jan;29(1):1-23. 